\title{
The gerB region of the Bacillus subtilis 168 chromosome encodes a homologue of the gerA spore germination operon
}

\author{
Bernard M. Corfe, ${ }^{1,2}$ Rachel L. Sammons, ${ }^{3}$ Derek A. Smith ${ }^{1}$ \\ and Catherine Mauël ${ }^{4}$
}

\begin{abstract}
Author for correspondence: Bernard M. Corfe (Krebs Institute). Tel: +44 742824418 . Fax: +44 7427286967 e-mail: b.m.corfe@sheffield.ac.uk
\end{abstract}

\author{
1 School of Biological \\ Sciences, University of \\ Birmingham, PO Box 363 , \\ Birmingham B15 2TT, UK \\ 2 Krebs Institute, University \\ of Sheffield, Sheffield \\ S10 2TN, UK \\ 3 Biomaterials Unit, School \\ of Dentistry, St Chad's \\ Queensway, Birmingham \\ B4 6NN, UK \\ 4 Institut de Génétique et de \\ Biologie Microbiennes, \\ Université de Lausanne, \\ Lausanne 1005, \\ Switzerland
}

\begin{abstract}
Spores of gerB spore germination mutants of Bacillus subtilis 168 are defective in response to the germinative mixture of L-asparagine, glucose, fructose and potassium ions (AGFK), but are normal in the L-alanine (ALA) triggered germination response. $A \lambda$ clone of $15 \mathrm{kbp}$ carrying the ger $B$ region has been identified. Sequencing of the ger $B$ region of the clone revealed a cluster of three ORFs encoding putative proteins of 53.3, 41.3 and 42.4 kDa (GerBA, GerBB and GerBC, respectively). The first two of these proteins have substantial hydrophobic regions and the third is a possible lipoprotein. At least two, and probably all three products are required for normal germination in AGFK. The three proteins form a set of homologues of the products of the gerA operon, mutations in which cause a defect in the ALA germination pathway, but cause no defect in AGFK. The GerB proteins show $42 \%, 31 \%$ and $35 \%$ identity at the amino-acid level to the corresponding GerA proteins, and the homologues occur in the same order in both operons.
\end{abstract}

Keywords: Bacillus subtilis, sporulation, germination, gerB, membrane protein

\section{INTRODUCTION}

Spores of Bacillus subtilis 168 have two known germination reponses, which have been well-characterized: they germinate in response to alanine alone (ALA) or to the mixture of asparagine, glucose, fructose and potassium ions (AGFK). Spore germination (ger) mutants have been isolated which are defective in either or both of these responses (Moir \& Smith, 1990), which suggests that there are separate germinant-specific triggers, with a later convergence of the germination pathways.

Of all the ger mutants the only ones with a specific defect in ALA germination result from mutation in the ger $A$ operon (Moir \& Smith, 1990). This is a tricistronic operon which encodes proteins of $53 \cdot 3,41 \cdot 3$ and $42.4 \mathrm{kDa}$, all of which could be membrane-associated (Feavers $e t$ al., 1985; Zuberi et al., 1987). The ger $A$ operon is expressed in the forespore compartment of the developing sporangium

\footnotetext{
Abbreviations: ALA, L-alanine; AGFK, L-asparagine; glucose, fructose and $\mathrm{K}^{+}$ions (germinative mixture).

The GenBank accession number for the nucleotide sequence reported in this paper is L16960.
}

from a promoter recognized by $\mathrm{E} \sigma^{\mathrm{G}}$, and it has been suggested that the proteins form a complex at the inner spore membrane which acts as a receptor for alanine (Feavers et al., 1990).

Mutants of gerB and gerK have defects specifically in the AGFK response but retain a normal ALA response (Moir et al., 1979; Irie et al., 1982) and gerK has been associated with the glucose component of the response on account of the inability of glucose to improve the ALA response in gerK mutants under certain conditions (Irie et al., 1982). This effect is not seen in gerB mutant spores (McCann, 1989). Mutants of gerD appear to be defective in both germination pathways to some extent (Moir et al., 1979); however the product of gerD may be associated with the fructose component of the response (Irie et al., 1986). The ger $B$ mutations have been located by phage PBS1-mediated three-factor transductional crosses to 314 degrees on the $B$. subtilis 168 chromosome. Mutations are not known to affect any one specific component of the AGFK response.

Molecular genetical analysis of several ger genes has as yet shed little light on the possible mechanism of germination (Moir \& Smith, 1990). The predicted proteins generally 
lack characteristic motifs and database searches reveal no homology with other proteins.

Although tag (teichoic acid biosynthesis) and gerB have not been mapped relative to one another, available data place them near to each other on the chromosome, with gerB on the ori-proximal side (Piggot et al., 1990). We have identified a $\lambda \mathrm{EMBL} 4$ derivative carrying ger $B$ from a series of overlapping clones of the tag-gta region. Sequence information indicating the possible nature of the gene products, reported here, has provided more essential information in the attempt to identify the mechanism of spore germination.

\section{METHODS}

Bacterial strains and media. Strains of B. subtilis 168 and Escherichia coli $\mathrm{K} 12$ used are listed in Table 1. B. subtilis was cultured on nutrient agar (NA), and E. coli on Luria-Bertani agar (LA), with appropriate selective conditions as described by Yon et al. (1989). Transformation of B. subtilis was by the method of Anagnostopoulos \& Spizizen (1961) and of E. coli by the method of Mandel \& Higa (1970). Ger phenotypes were scored on plate tests as described by Irie et al. (1982).

DNA manipulation. Plasmid and chromosomal DNA preparation, restriction, ligation and gel electrophoresis were carried out as described by Sammons et al. (1987). All subcloning was done using pMTL20EC in DH5 $\alpha$. $\lambda$ DNA preparation was as described by Maniatis $e$ t al. (1982), using E. coli $\mathrm{P} 2392$ as a host. Purification of restriction fragments from gels was achieved using Geneclean (Bio101).

DNA sequencing. Restriction fragments of $150-1500$ bp were

Table 1. Bacterial strains

\begin{tabular}{|c|c|c|}
\hline Strain & Genotype & Source \\
\hline \multicolumn{3}{|c|}{ B. subtilis } \\
\hline L.5047 & $\begin{array}{l}\text { phe } A 1 \text { pur } A 16 \text { bis } A 35 \text { trpC } 2 \\
\text { metB5 }\end{array}$ & D. Karamata \\
\hline 1604 & $\operatorname{trp} C 2$ & Lab. stock \\
\hline 4688 & $\operatorname{gerB18} \operatorname{trp} C 2$ & Lab. stock \\
\hline 4950 & $\operatorname{gerB} 90 \operatorname{trp} C 2$ & Lab. stock \\
\hline 4952 & gerB92 trpC2 & Lab. stock \\
\hline 4954 & gerB94 trpC2 & Lab. stock \\
\hline 5182 & gerB15 $\operatorname{trp} C 2$ & Lab. stock \\
\hline 5301 & $\begin{array}{l}\text { gerB18 phe } A 1 \text { pur } A 16 \text { bis } A 35 \\
\quad \operatorname{trp} C 2\end{array}$ & This study \\
\hline 5304 & $\begin{array}{l}\text { gerB15 phe } A 1 \text { pur } A 16 \text { bis.A35 } \\
\quad \operatorname{trp} C 2\end{array}$ & This study \\
\hline \multicolumn{3}{|l|}{ E. coli } \\
\hline $\mathrm{DH} 5 \alpha$ & $\begin{array}{l}\mathrm{F}^{-} \text {end } A 1 \text { bsd } \mathrm{R} 17\left(\mathrm{r}^{-} \mathrm{m}^{+}\right) \text {sup E44 } \\
\text { thi-1 } \operatorname{rec} A 1 \text { gyr } A 96 \text { relA1 } \\
\Delta(\operatorname{argF}-\operatorname{lac} Z Y A) \cup 169 \phi 80 \mathrm{~d} \\
\text { lac } Z \Delta M 15\end{array}$ & $\begin{array}{l}\text { D. Karamata } \\
\text { (Lausanne) }\end{array}$ \\
\hline P2392 & $\begin{array}{l}\mathrm{F}^{-} \text {bsdR514 supE44 supF58 lacY1 } \\
\text { galK2 galT22 metB1 trpR55 } \\
\text { lysogenic for } \mathrm{P} 2\end{array}$ & D. Karamata \\
\hline
\end{tabular}

prepared from parent subclones, gel-purified, subcloned into pMTL20EC and sequenced. M13 universal, reverse and -40 primers were used wherever possible, supplemented by a small number of custom primers. Double-stranded dideoxy sequencing was done using the Sequenase system as recommended by the manufacturers (USB). Electrophoresis was carried out using $4-8 \%(\mathrm{w} / \mathrm{v})$ denaturing acrylamide gels in a BRL S0 apparatus (Gibco).

\section{RESULTS}

\section{Identification of a gerB clone and sequencing}

Mauel et al. (1989) identified clones carrying the tag region in a $\lambda$ EMBL3 bank and Young et al. (1989) subsequently extended the cloned region by chromosome-walking using $\lambda E M B L 3$ and $\lambda E M B L 4$ banks. As ger $B$ was thought to be on the ori-proximal side of $t a g$, the appropriate clone, $\lambda E 51$, was screened for the presence of gerB. As $\mathrm{Ger}^{+}$ phenotypes cannot be selected directly, ger $B$ phe $A$ derivatives of L5047 were made for screening purposes. DNA from strains carrying either the gerB15 or the gerB18 allele, 5182 and 4688, respectively, was used to transform strain L5047. Selection was for $\mathrm{Met}^{+}$and transformants were screened to find strains with a $\mathrm{Ger}^{-}$phenotype. Appropriate double mutants, strains 5304 and 5301, were transformed with a mixture of $0.5 \mu \mathrm{g}$ DNA from a $\lambda$ clone carrying $\mathrm{Phe}^{+} \mathrm{DNA}$ and $5 \mu \mathrm{g} \lambda \mathrm{E} 51$. One hundred and fifty transformants from each cross were screened for their Ger phenotype using a modification of the tetrazolium test (Irie et al., 1982) and it was found that in congression experiments with the $\lambda E 51$ DNA present both the ger $B$ alleles were corrected, with about $50 \%$ of the $\mathrm{Phe}^{+}$ transformants having a $\mathrm{Ger}^{+}$phenotype. Congression experiments with $\lambda \mathrm{E} 51$-derived plasmid subclones in place of $\lambda E 51$ revealed the approximate location of two of the ger B alleles within the clone (Fig. 1).

The sequence of the ger $B$ region was generated by dideoxy sequencing of small restriction fragments subcloned in plasmid vector pMTL20EC (Chambers et al., 1988) as described in Methods. All restriction sites were overlapped in the sequence.

\section{Computer analysis of the sequence}

The sequence of $3.8 \mathrm{~kb}$ was analysed using the UWGCG package, version 7.1 (Devereux et al., 1984) except where otherwise stated. Three large adjacent ORFs were identified by direct translation and were confirmed by codon usage analysis (data not shown). The sequence and putative translations are shown in Fig. 2. ORFs 1, 2 and 3 have been named $\operatorname{ger} B A$, ger $B B$ and $\operatorname{ger} B C$, respectively. There are three possible initiation (start) codons for ger $B B$, a GTG and ATG which overlap the gerB $A$ termination (stop) codon, and an ATG which is 5 bp downstream. The last has a possible Shine-Dalgarno sequence, although it is weak and close to the ATG. There is an overlap between the probable stop and start codons of ger $B B$ and ger $B C$. All the ORFs have potential ribosomebinding sites with homology to the $3^{\prime}$ end of the B. subtilis 16S RNA molecule (Stewart \& Bott, 1983) appropriately 


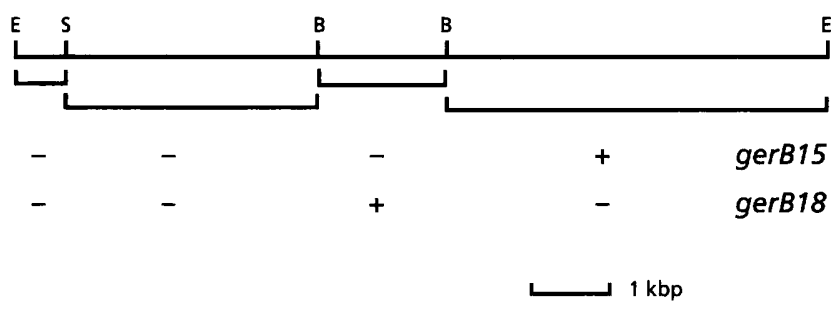

Fig. 1. Marker correction with $\lambda E 51$. Restriction map of the insert in $\lambda E 51$ and four plasmid subclones derived from it. The five clones were used in congression experiments (see text) to localize the position of the gerB15 and gerB18 alleles in the insert. B, BamHI; E, EcoRI; S, Sphl; +, correction to Ger'; -, no correction of Ger phenotype.

located in front of their start codons (see Fig. 2). This arrangement suggests that the ger $B$ mutations may identify an operon. There is no obvious terminator after $\operatorname{ger} B C$, but no start of another ORF.

The predicted molecular masses of GerBA, GerBB and GerBC are $53.971,41.709$ and $42.468 \mathrm{kDa}$, respectively. Database searches using the UWGCG Fasta program revealed extensive homology between the polypeptides encoded by ger $B$ and those encoded by the ger $A$ operon as shown in UWGCG Gap comparisons (Fig. $3 a-c$ ). The homology exists across the full length of the genes' products and all the pairs of proteins are of similar size. GerBA and GerAA are $42 \%$ identical at the amino acid level, and show $66 \%$ similarity, taking conservative substitutions into account, of the primary sequence. Hydroplots (Kyte \& Doolittle, 1982) show a central hydrophobic domain sandwiched between a large hydrophilic domain at the $\mathrm{N}$-terminus and a smaller one at the C-terminus of GerBA (Fig. 4a). The polypeptides are well-conserved at the functional level, but the hydrophobic domain shows the greatest level of identity. This is unusual, as hydrophobic regions are generally conserved for functionality rather than identity (e.g. Hiles et al., 1987; Higgins et al., 1990).

A further possible member of the GerAA/GerBA group is the spoV $A F$ gene product. This ORF was partially sequenced by Fort \& Errington (1985) as the sixth predicted member of the spoV $A$ operon and the predicted $\mathrm{N}$-terminal sequence had homology to GerAA. When the sequence of the downstream gene, lys $A$, was published, the upstream sequence included the second half of spoVAF (Yamamoto et al., 1991; Sorokin et al., 1993) so that the complete sequence is now available. UWGCG Gap comparisons of GerAA and GerBA with the predicted SpoVAF polypeptide showed a lower degree of conservation than exists between GerAA and GerBA (data not shown), although the polypeptide is still significantly homologous, with around $25 \%$ identical residues and over $50 \%$ similarity taking conservative substitutions into account. SpoVAF is also of a similar size (462 amino acid residues) to GerAA and GerBA.
However, insertional inactivation of spoV $A F$ has no known effect upon spore germination (E. H. Kemp, personal communication). There are no further ORFs downstream of spoV $A F$ in the putative operon.

The hydroplot of GerBB (Fig. 4b) shows that it is predominantly hydrophobic with no large hydrophilic regions. There is homology across the whole length of the GerAB/GerBB polypeptides but, as is common for hydrophobic proteins, this is at the functional $(60 \%$ conservative substitutions) rather than identical $(30 \%$ identity) level.

The hydrophobic domains of both GerBA and GerBB are similar in size to their GerA homologues, but their hydroplots are not as clearly defined as for GerAA and GerAB, making estimation of the number of membranespanning helices difficult on the basis of these data. However the close similarity with the ger $A$ products, and the clarity of the hydroplots of the latter, could suggest that GerBA and GerBB have seven and 10 or 11 transmembrane regions, respectively. If this is correct then the hydrophilic domains of GerBA would be on opposite sides of the membrane.

GerBC is predominantly hydrophilic, but has a small hydrophobic region at the $\mathrm{N}$-terminus which has homology to the signal sequence for exported prokaryotic lipoproteins (Yamaguchi et al., 1988). GerBC shows $35 \%$ identity with its homologue, and $58 \%$ conservative substitution.

Upstream of the $\operatorname{ger} B A$ translational start is a potential binding site for $\sigma^{\mathrm{G}}$, the sigma factor which directs gene expression in the forespore after engulfment by the mother cell (Fajardo-Cavazos et al., 1991). This may indicate a further degree of homology between the two gene clusters, as the ger $A$ operon is a known member of the $\sigma^{\mathrm{G}}$ regulon (Feavers et al., 1990). Between bases 10 and 20 of this sequence is the -35 region for ORFX, a putative gene in the opposite orientation to gerB in the upstream sequence (Margot, 1992). ORFX lies adjacent to lytD (glucosaminidase) which in turn is adjacent to the tag $A B C$ operon (Margot, 1992).

\section{Localization of the gerB alleles}

Strains carrying ger $B$ alleles were transformed with integrative plasmids carrying regions of the putative ger $B$ operon. Three subclones were used: A carried most of $\operatorname{ger} B A$, and the upstream region; $\mathrm{B}$ carried an overlap between $\operatorname{ger} B A$ and $\operatorname{ger} B B$; and $C$ carried an overlap between ger $B B$ and ger $B C$. The results of the integrations are shown in Fig. 5. The integration of subclone $C$ into wild-type gives a TZM ${ }^{\mathrm{W}}\left(\mathrm{Ger}^{-}\right)$phenotype. This indicates that the ger $B C$ product must be required for germination. This result for subclone $C$ is an argument for operon structure. There is a complete copy of the gerBC ORF on the chromosome after the integration event, but a $\mathrm{Ger}^{-}$ phenotype is obtained. Therefore the ORF must be separated from its promoter by at least the $250 \mathrm{bp}$ which are present between the start of the subclone and the start 
HindIII

1 AAGCTTTGTTTCAGCCTTTTCCTCGATAAGAATAATTCTCCTTTTTTGA TACAAATTAATAAAAACCGTCAATATGTTTAAGAAGGAGAGAAACTATGCA

101 AATCGACTCTGATCTCCAGÄACAATTTAGACACACTGAAAAAAACATTGGGACAAAACGACGATATGATGTTTTATACATTTGCTTTCGGAGATAGCAGÁ

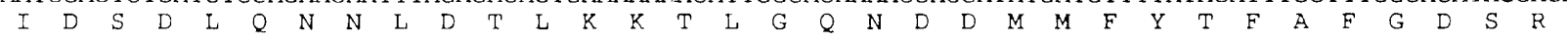

201 CAAAAGGCGTGTTTACTGTÁmATTGACGGTCTGACAGAGAATAAAATGCTGGCGCAATACGTCATTTCTCCTTMACAAAAAGAGGCGTTGGCCCATAAGG

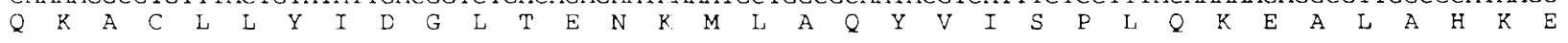

301 AATGCTCGATTGAAGACTTÁTCCGCCTTMTTTTTCGGCTTTCACCACAGCGTTGTTTCTACAATGAAAGAAATAGAGCAGCTCGTTTTTTCAGGGCAAGC

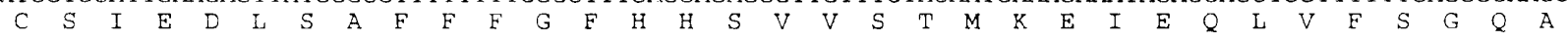

401 CATTTTGCTCGCTGATGGCTATCGCGGAGGATTAGCTTTCGAC ACAAAGTCAGTCGCAACACGCAGTCTTGATGAACCCTCCTCCGAAGTCGTGGAACGC

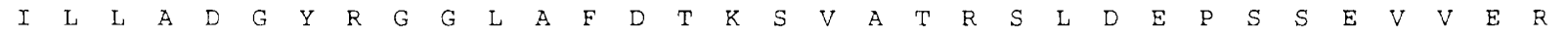

501 GGCCCTAAAATAGGATTTATTGAAAAGCTGGGGACAAACACGGCGCTTTTGCGTGAACGGACGAGTGATCCTAATCTCGTCATCAAAGAAATGACACTCG

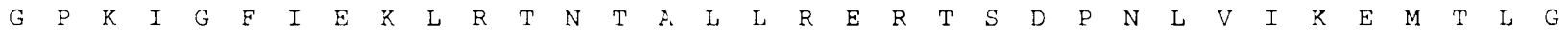

601 GAAAAAGGACCAAAAAGAAAATCGCTGTCGCTTATATTCAGGATATCGCCCCAGATTATGTCGTCAAAGAAGTGTTTAAAAGGCTGAAATCAGTCAATAT

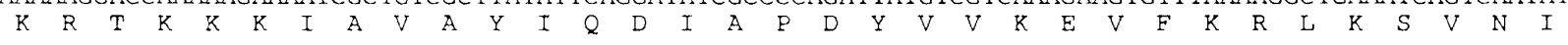

701 AgATAATTTGCCGGAATCGGGGACGCTTGAACAGCTGATTGAдGACGAACCATTTTCAATTTTCCCCACAATATTAAGCACAGAACGACCGGACCGGGTC

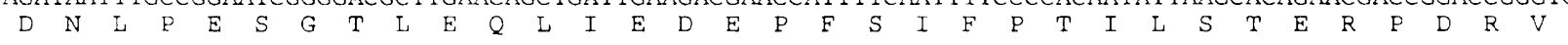

801 GAAAGTrCTCTATTAGAAGGGCGAGTGTCCATATTAGTGGATGGTACGCCGTTTGCTTTGATTGTACCGGCTACGGTTGACGAATTTATTCATTCGCCCG

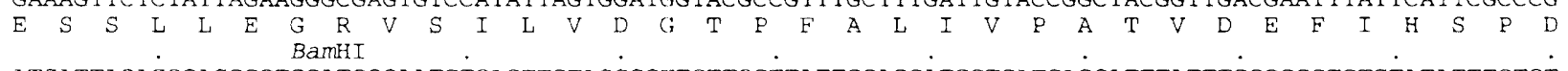

901 ATGATTACAGCCAGCGCTGGATCCCAATGTCACTTGTACGCC.TCTTCGTMATCCAGCATCCTGATCACCATTTATTTGCCCGGTCTGTATATTTCTCT

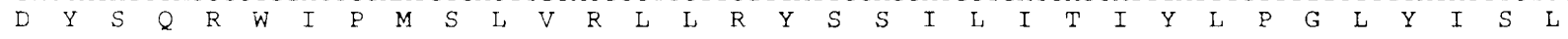

1001 CGTTRCTTTTCACACCGGGCTATTGCCGACTAGAATGGCCATITCCATTGCAGGCAGCAGGCTTAACGTACCATTCCCGCCTTTTGTAGAGGCCTTMATC

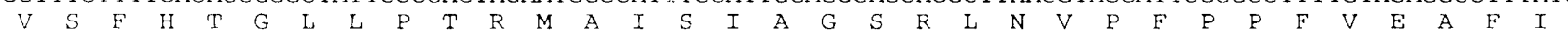

1101 ATGATCTTTÁCGATCGAATTGATTCGAGAAGCCGGATTAAGGCTGCCTAAGCCGATTGGACAGACAATTGGCCTTATCGGCGGCGTTGTCATCGGACAGG

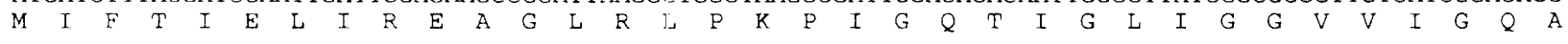

1201 CAGCTGTTCAGGCGCAAATTGTCAGTGCGCTTATGGTCATTGTCGTTTCTGTCACAGCACTGGCATCCTTTACCGTTCCTTCATACGCCTATAACTTTCC

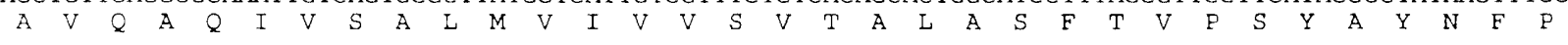

1301 GCTGCGGATCATTCGGATCGGGGTPATGATAAGTGCAACAGCGCTTGGCATGTACGGCGTTATAATGGTTTATCTGTTMGTGATCGGCCATCTCATGCGC

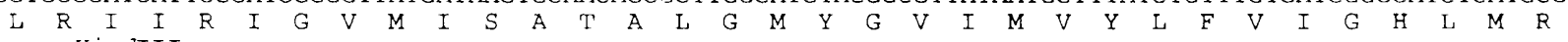
HindIII

1401 CTGAAAAGCTTGGCCAGGATTACATTATCCGATCATGCGCAGCCTGGACAGGATTTGAAAGACACAGTCATCCGTATTCCCACGATGTTITTAAAAAGA.

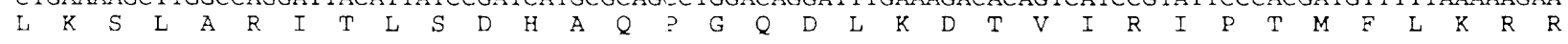

1501 GACCGACACGAAACGATCCCGAAGATAACATCAGACAAAGGTZATGGCTÁTGAGGAAATCAGAGCATAAACTGACATTTÁTGCAGACGCTCATTATGATC

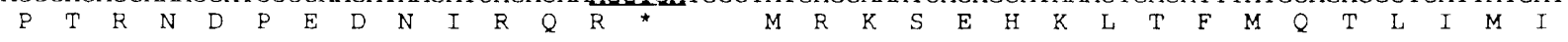

1601 AGCAGCACATTGATTGGTGCCGGGGTGCTGACCCTTCCCCGCIPAGCCGCCGAAACCGGCAGTCCGAGCGGATGGCTAATGATACTGCTCCAGGGCGTTA

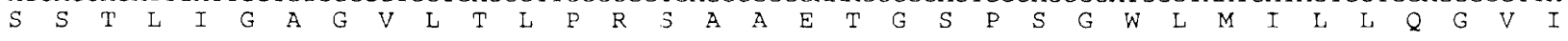

1701 TTTTTATTATCATCGTTCTGCTTTTTTTGCCTTTTCTTCAAAAAAACAGCGGAAAAACTCTTTTTAAGCTCAACAGCATTGTAGCTGGGAAATTCATCGG

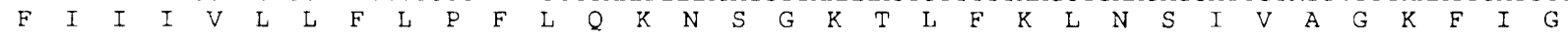

1801 CTMTCTATTGAATTTATATATCTGTCTATATTTCATTGGGATTGTTTGCTTTCAAGCTCGGATTTTGGGAGAGGTTGTCGGATTCTTTTTGTTGAAAAAT

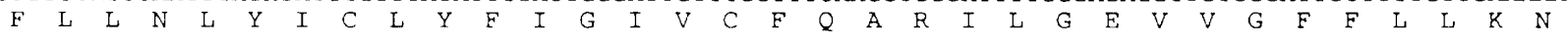

1901 ACCCGAATGGCAGTTGTGGTGTTTATATTTCTTGCAGTTGCCATCTATCÁTGTAGGCGGAGGCGTTTATTCAATTGCAAAAGTATACGCT'TATATTTTTC

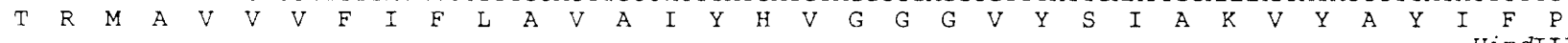

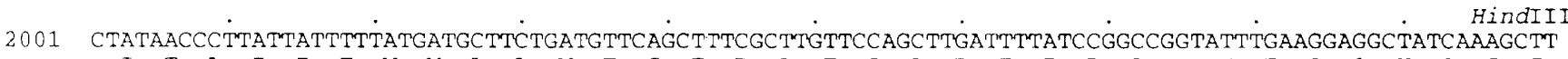

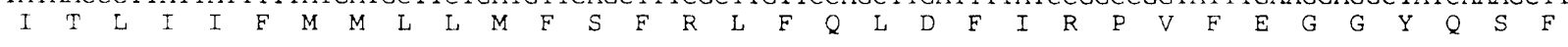

2101 TTTCTCTTPATTCCCAAAAACATTATTATATTTCTCCGGATTTGAAATCÁTTTTTTACCTGGTCCCCTTTATGAGAGATCCAAAGCAAGTGAAAAAGGCT

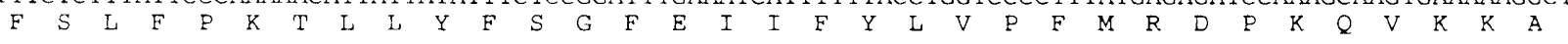

2201 GTTGCTCTGGGCATCGCGACTTCCACATTGTTCTACAGCÁtTACTTTGCTCATTGTGATTGGCTGTATGACTGTGGCTGÄGGCAAAAACGGTGACATGGC

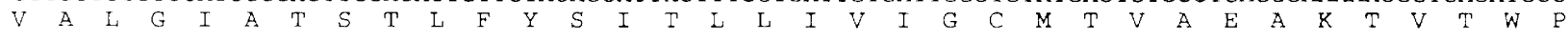

2301 CGACCATTTCCCTTATTCACGCATTAGAGGTTCCGGGTATTTTTATTGAACGATTTGATTTGTTCTTACAGCTGACCTGGACAGCCCAGCAATTTGCCTG

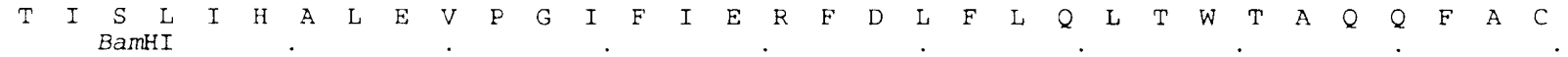

2401 TATGCTCGGATCCTTTAAAGGCGCCCATATCGGGTTAACGGAAATCTTTCATCTAAAAAATAAAAACAATGCATGGCTGCTGACTGCAATGCTGGCTGCG

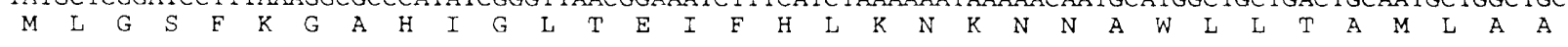

2501 ACGTTTTTCATAACGATGTÁTCCCAAGGACTTGAATGACGTGTTTTATTACGGGACGCTTCTTGGATATGCTTTCTTAATTGTGATTACAATTCCATTCT

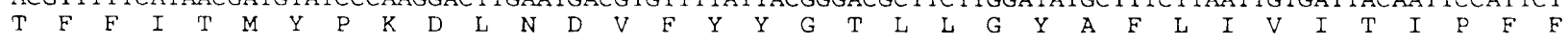

2601 TCGTCTGGTPTTTAAGCTGGATACAGAAAAAAATAGGGAGGGGGCAGCTGCAATGAAAACAGCATCAAAATTCTCCGTCATGI'TMTTTATGCTGCTGGCC $V \quad W \quad F \quad L \quad S \quad W \quad I \quad Q \quad K \quad K \quad I \quad G \quad R \quad G \quad Q \quad L \quad Q$ * $\begin{array}{lllllllllllllllllllllllll}M & K & T & A & S & K & F & S & V & M & F & F & M & L & L & A\end{array}$

2701 CTCTGCGGCTGCTGGgATGTCAAAGATATCGAGCAACTATCC TTCGCCAGAGGGCTGGCGATCGATGAGACAAATGATCACCAGTACAAACTGACGTATC

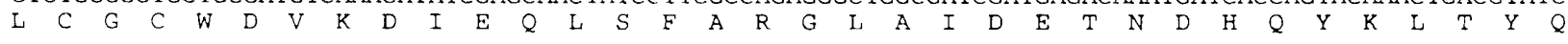


2801 AAAATCTTCTTCCCCAAAGCGAAGACAGCCAAGCATCCGGAAAACCCGAं̈TTTGTTAATGTAACGTCCCÄGGGAAAACGATTCTGGAAGCAGTCAGTGA்

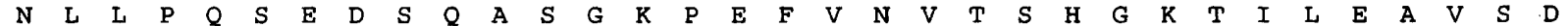

2901 TGTATCCATCAAGGACCCTCCTGTTTATAG்GATCATTTAAAAGTGATCCंTTCTGGGAGAAAAACTGATGAGGAATCAAAATGTAGACCAAGTCCTCAAC $\begin{array}{lllllllllllllllllllllllllllllllllll}V & S & I & K & D & P & P & V & Y & S & D & H & L & K & V & I & L & L & G & E & K & L & M & R & N & Q & N & V & D & Q & V & L & N\end{array}$

3001 CACTTTATCCGGGATGATGÄGCTGCGGCGÄGCAGCTATTTGATGGCAGCCAGAGGGAATGCAGCTGATGTTTTCACAAAAGGAAACCCCAATCAGCAGCं $\begin{array}{llllllllllllllllllllllllllllllllllll}H & F & I & R & D & D & E & \text { L } & R & R & S & S & Y & L & M & A & A & R & G & N & A & A & D & V & F & T & K & G & N & P & N & Q & Q & Q\end{array}$

3101 AGCCGATGCCCTCCGAAAAACTGATTGATTTGACAACTCÁCAGCGGATATAACGGTAAGAंTTATGATACCGCTGCGCATCGGAAGAGCTTCTGTCTACTC

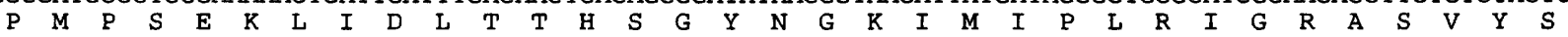
TCAGAACGGGTACAGTTATCTTATTCAAGCCGTGAAAAACGAAAAGGGAAAAGCCAAGTÁTGACGGAGCÁGGCATTATCAAAAGGGGCAGCAATAAACTC

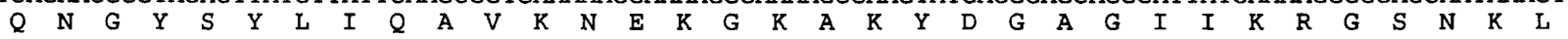

3301 GTTGGGTTTCTTTCAGCTGÄTGAAACCCAÄACACTGTCATGGGTCATGGGGACGATCCAÄGGGGTGTCÄTGCCGACGACAGATAAAGGACATCCGATTA

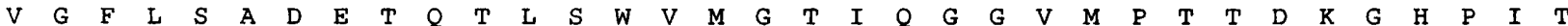

3401 CGTTTGAAATTAAAAAGTCGAAAACGAAAAंTTAAGCCCGTCATTGAAAACGGAAAGCCTǴTCTTTCATATTTCTGTTAAAACGAAGGGCAंTCCTGACAGA $\begin{array}{lllllllllllllllllllllllllllllllllll}F & E & I & K & K & S & K & T & K & I & K & P & V & I & E & N & G & K & P & V & F & H & I & S & V & K & T & K & G & I & L & T & E\end{array}$ .HindIII

3501 AGACCAAAACCCGAATGAAAACTCCTTTAGCAAAAGTTATTTGCACAGGCंTGGAAAACATTTTTGAAAAAAAGCTTGAACGAGATGTAAAGCAGGTGATG

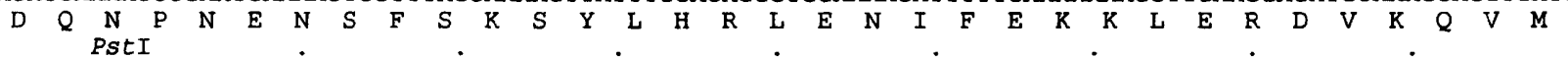
PStI 3601 GATAAACTGCAGCACGAGTATAAAACCGATCCGGTCTTTTTATCAGACCACATAAGGATTCAACACCCTGACTACTGGAATAAAGTAAAAGGGCATTGGG

3701 ATGAAATATTTTCTGAGACTGATTTTAAGTACGATATTTCंTTTTAAAATCATTAACTTTGGCACGGTGGGAAAGTAAGCAATCAAAAGGAGCGCGTGATC $\begin{array}{lllllllllllllllllllllllllll}E & I & F & S & E & T & D & F & K & Y & D & I & S & F & K & I & I & N & F & G & T & V & G & K\end{array}$

3801 GCACCCTTTTTATGTTCCGACAGTCTGCT 3829

Fig. 2. Nucleotide sequence of the ger $B$ region. The sequence of $3829 \mathrm{bp}$ is derived from subclones shown in Fig. 1. There are three large ORFs which are shown below the sequence, each with a potential ribosome-binding site which is underlined. The operon also has a potential $\mathrm{E} \sigma^{\mathrm{G}}$-type promoter upstream of ORF 1 , which is double-underlined. There is no obvious terminator after ORF 3.

of the ORF. The integration of subclone $B$ into the wildtype gave a $\mathrm{Ger}^{+}$phenotype. This result may be misleading, as there is a possibility of readthrough from a plasmid-borne promoter with the vector, pMTL20EC, which may have yielded sufficient gene product to give the $\mathrm{Ger}^{+}$phenotype. This would not affect the result with $\mathrm{C}$, as the ORF and plasmid are in the opposite relative orientation in this construct. A plasmid construct was made with DNA from subclone B cloned in the same vector in the opposite orientation. When integrated into strain 1604 this plasmid gave a $\mathrm{Ger}^{+}$phenotype, which is a further argument for operon structure. Whilst readthrough from a plasmid promoter at sporulation may only give a low level of expression, this may be sufficient. The level of ger $A$ expression is known to be low (Feavers $e t$ al., 1990 ) and multiple promoterless copies of ger $A$ ORFs are sufficient to complement ger $A$ mutations (Zuberi et al., 1985).

The pattern of results for plasmid integration into the mutant strains provides information as to the locations of the ger $B$ alleles. The data show that the gerB90, -92 and -94 alleles all lie in $\operatorname{ger} B A$ as they are all corrected by subclone $\mathrm{A}$, which only carries DNA from the ger $B A$ region, but the alleles have distinct locations within the ORF as ger $\mathrm{B} 90$ and -94 are corrected by subclone $\mathrm{B}$, which overlaps this region. As gerB18 is not corrected by A but is corrected by $\mathrm{B}$ it can be deduced that the lesion lies in the last 50 codons of $\operatorname{ger} B A$ or in the first 296 codons of $\operatorname{ger} B B$. Hence $\operatorname{ger} B A$ and $\operatorname{ger} B C$ have been shown to be required for AGFK germination, and it seems likely that ger $B B$, too, is necessary.

\section{DISCUSSION}

The sequence of the ger $B$ spore germination operon has been elucidated and has revealed that $\operatorname{ger} B$ is a homologue of the ger $A$ operon. In addition spoV $V A$, the sixth ORF in the spoV $A$ operon (Fort \& Errington, 1985), has a lower degree of homology with the ger $B A / \operatorname{ger} A A$ group. Furthermore ger $K$, mutations in which cause a defect in the AGFK response, has been shown to encode a further homologue of the GerAC/GerBC group (R. Irie, unpublished). GerK is more closely related to GerBC than to GerAC, but is not as close to either as they are to each other (B. Corfe, unpublished). Hence a family of proteins has now been identified, mostly involved in spore germination and produced in the forespore. Mutations affecting germination have been mapped in all three ORFs of the ger $A$ operon and in at least two of the ger $B$ operon (see Fig. 5), demonstrating the requirement of all these proteins for normal germination.

The highly conserved hydrophobic region in the GerAA/ GerBA group probably indicates a region of functional importance. It is unlikely that this indicates a recent evolutionary divergence as the rest of the homology shows more divergence in both the hydrophobic and hydrophilic domains. Furthermore such a high level of identity between two hydrophobic domains that have not 
(a)

GeTAA 1 MEQTEFKEYIHDNLALVLPKLKENDDLVKNKKKMLANG. LVFYYLYFSEMT 49

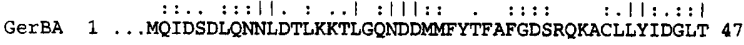
50 DENKVSEAIKTLIKDEETL, ... TLDQVKKKRLDQLDARPVETAKKTIESI 95 48 ENKMLAOYVISPLQKEALAHKECSIEDLSAFFFGEHHSVISTMKEIEQLV 97 96 LNGNCAVFINGLDKAYILTTGKKKTRSLTEPTTEKVVRGPKVAFVEDIDT 145 98 FSGQAILLADGYRGGLAFDTKSVATRSLDEPSSEVVERGPKIGFIEKLRT 147 146 NLALIRQRTSंHPKLITKKIMIGENKLKPAAंIMYIEGKAKKSVIKEVKARI 195

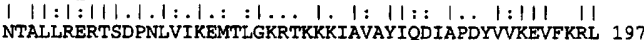
196 KNIQLEDIQDSSGTLEELIEDNKYSPFPQIQNNTERPDKVSSALFNGRVAIL 245 198 KSVNIDNLPESGTLELIEDEPFSIFPTILSTERPDRVESLLEGRVSIL 247 246 VDSSPFVLLVVVSLGILMQSPDDYYERWISASLIRSLRFASIFITLFLSS 295

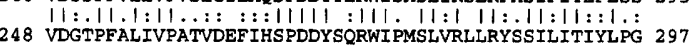
296 IYIALVSFHQGLLPTALAVTISANRENVPFPPIFEALLMEVTIELLREAG 345

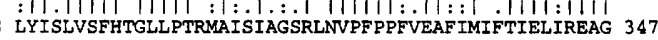
346 ..LPNPLGQTIIGLVGGVIGQAAVEANLVSSILVIVVSVIALASFTVPQY 393

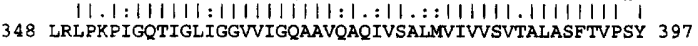
394 GMGLSFRVLRF ISMFSAAILLLYGI ILFMLVVYTHLTRQTSFGSPYFSPN 443 398 AYNFPLRIIRIGVMISATALGMYGIMVYFVIGHLMRLKSLARITLSDH 447 444 GFFS. .LKNTDDSIIRLPIKNKPKEVNNPNEPKTDSTET 480

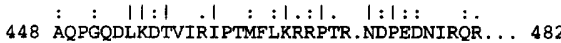

(b)

GeTAB 1 MSQKQTPLKLNTTFQGISIVANTMLGAGLLTLPRALTTKANTDGWITLILE 50 GerBB 1 . MRKSEHKLTFMQTLIMISSTLIGAGVTTLPRSAETGSPSGWLMILL 48 51 GFIFIFFIYLNTLIQKKHQYPSLFEYLKEGLGKWIGSIIGLLICGYFLGV 100 9 GVIFIIIVLLFLPFLQKNSGKTLFKLNSIVAGKFIGFLLNLYICLYFIGI 98

101 ASFETRAMAEMVKFFLLERTPIQVIILTFICCGTYLMVGGLSDVSRLFPF 150

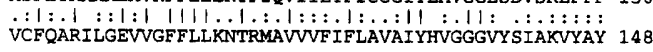

151 YLTVTIIILLIVFGISFKIFDINNLRPVLGEGLGPIANSLTVVSISFLGM 200 149 IFPITLIIFMMLLMFSFRLFQLDFIRPVFEGGYQ: : $:$ : $:$ : $:$ : 1 I $1: 19$ 201 EVMLFLPEHMKKKKYTFRYASLGFLIPIILLFLTYIIVVGALTAPEVKTL 250

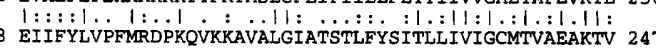

251 IWPTISLFQSEELKGIFIERFESFLLVWWIIQEFTTFVIYGGYFAANGLKK 300

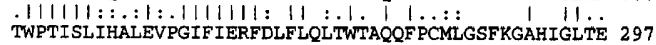

301 TFGLSTKTS... MVIIGITVYFYFSLWPDDANQVMMYSDYLGGIFVSLFLL 347 $\begin{array}{llll}1 & 1 \ldots \\ \text { IFHLKNKNAWLLTAMLAATFFITMYPKDLNDVFYYGTLLGYAFLIVITI } & 347\end{array}$

348 AVHSLFHVALKRRITTTK. . . 364

348 PFFVWFLSWIQKKIGRGQLO 367

(c)

GerAC 1 MKI..RILCMFICTLLLSGCंNDSENIEELSLVIGIGLDKPDDENLELTQQ 48

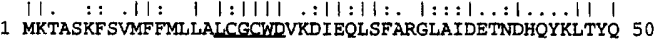

49 ILVPKIISAKEGSSSDPTQLSITK. GKTVHQMMRTSALKHKPTFSQHSRL 97

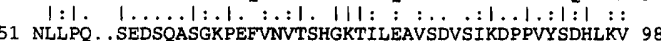

98 ILLSKSVIADQRIGMDAIINQEVVRDNGTRRSSSYVF ITNGRTKDIF . . NMN 144

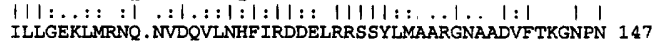

145 DEGEPASNVIYDLTENNKVTIRTME PVTLGEISEHLTSDDSFLIPHVGKE 194

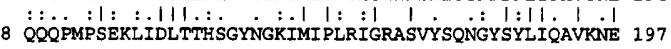

195 NGKLAINGASIIIK. . . NKLWHHRDLTPIEVQNISLFSGTVEGGVIDLKRDG 241

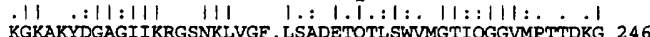

242 HLFSYEVYSSNRKIKTAYKDंGKFKFTVTRNIEGRLSEDWNPNEDSFKDSY 291

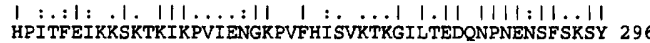

292 IKSIEKTVEKRVHETVTSFITTELQKEIKADDVTGLGNEVRIHYPQKWKKI 341 297 LHRLENIFEKKLERDVKQ. VMDKLQHEYKTDPVELSDHIRIQHPDYWNKV 345

342 SRKWDDDYF SNAEIDYRVNVIIVRDFGTKGAंNK 374

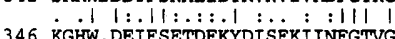

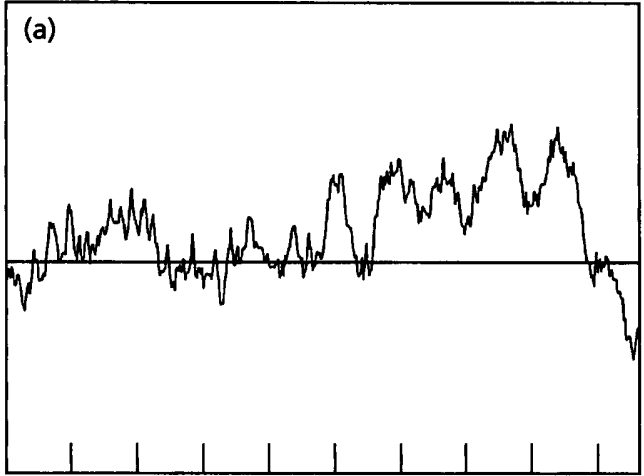

(b)

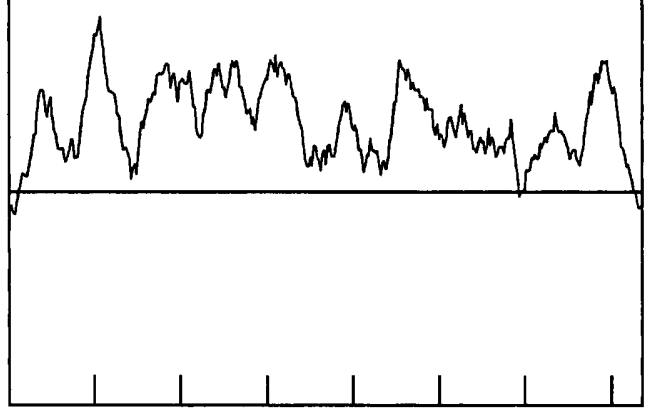

Fig. 4. Hydroplots of the GerBA and GerBB proteins. The $y$-axis shows increasing hydrophobicity, with the mean hydrophobicity for a large number of proteins represented by a horizontal line; the scale is from -4 to +4 , according to the algorithm of Kyte \& Doolittle (1982). Markers on the $x$-axes are at every 50 residues. The plots were calculated using a window size of 21. (a) The GerBA profile indicates an N-terminal hydrophilic domain, with possibly seven membrane-spanning hydrophobic segments and a smaller hydrophilic C-terminus. This pattern is similar to that found in GerAA. (b) The GerBB profile is highly hydrophobic, and is also very similar to its GerA counterpart. The pattern may suggest 10 or 11 transmembrane regions.

recently diverged is uncommon. This argues that this region of the protein must be particularly important in the function of the protein. As yet no possible functions have been individually assigned to any of the GerA/GerB proteins.

There are significant genetical and physiological differences between the ALA and AGFK germination responses. The germinant requirement is very different:

Fig. 3. Gap comparisons of the GerA and GerB proteins. The scores for percentage identity and conservative substitution for the pairs of proteins are as follows: $42 / 66$ for GerAA vs GerBA (a); $31 / 60$ for GerAB vs GerBB (b); 35/58 for GerAC vs GerBC (c). The most highly conserved region of the GerAAVGerBA pair is the hydrophobic domain between residues 200 and 400 . This pattern of conservation is absent in GerAB and GerBB, which are predominantly hydrophobic proteins. The GerAC and GerBC proteins have homology to the prokaryotic lipoprotein attachment motif (underlined). Solid lines represent identical residues, double dots represent strongly conservative substitutions, and single dots represent weakly conservative substitutions. 


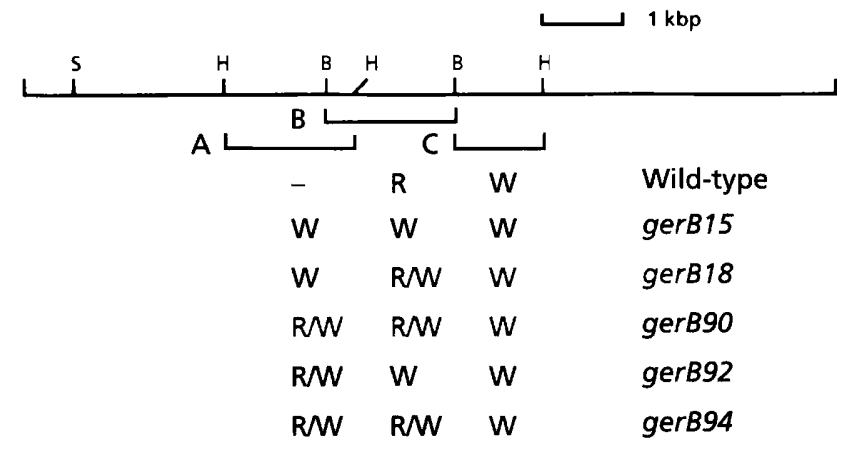

Fig. 5. Localization of the gerB alleles. The diagram shows the results of integration of three plasmid subclones into ger $B$ mutant strains and the wild-type. Integration of subclones $A$ or $B$ into mutant strains may give rise to a mixture of $\mathrm{Ger}^{+}$and $\mathrm{Ger}^{-}$phenotypes if the subclone can correct the allele, depending upon whether recombination takes place to one side or the other of the lesion. The pattern indicates that gerB90 and gerB94 both occur in the region of overlap between $A$ and $B$, gerB92 occurs in the region of $A$ not overlapped by $B$, and the converse for gerB18. Subclone $A$ covers all of ger $B A$ except for the last 50 codons. $R$, integrants all $\mathrm{Ger}^{+} ; \mathrm{W}$, integrants all Ger ; RM, mixture of $\mathrm{Ger}^{+}$and $\mathrm{Ger}^{-}$ phenotypes upon integration; B, BamHI; H, HindIII; S, Sphl.

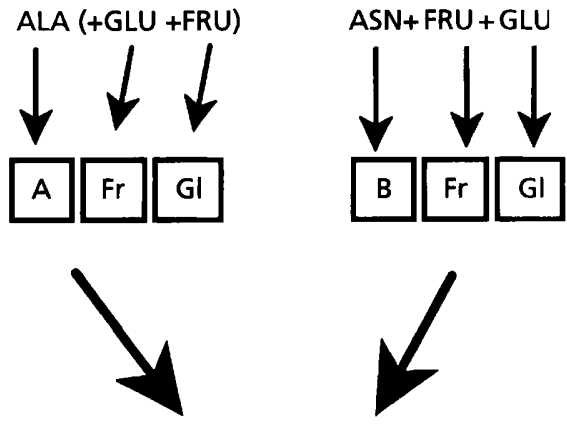

Germination

Fig. 6. Model for germinant receptors in the spore. The square boxes are ger-encoded proteins. The GerA-based receptor will respond to ALA alone, but the response will be improved by the presence of GLU and FRU stimulating the response via the appropriate gene products (which may be encoded by gerD and gerK). In the GerB-based receptor the additional gene products and germinants are obligatory for germination.

the ALA response depends upon a single amino acid as germinant (which is thought not to be metabolized, as non-metabolizable analogues of alanine will also trigger germination: Sammons et al., 1981), and the response is not affected by metabolic inhibitors. Mutations in $\operatorname{ger} A$ are the only ones known to specifically block the ALA response without affecting the AGFK response. In contrast a complex set of germinants is required for the AGFK response, the response is inhibited by sodium azide (Venkatasubramanian \& Johnstone, 1989) and mutants of ger $B$ and ger $K$ are specifically blocked in the response.
The ger $A$ products appear to form a complete receptor/ trigger unit in their own right, as the ger $A$ operon is the only known operon in which mutation results in failure of spores to respond specifically to alanine. The ger $B$ region encodes homologues of the complete ALA receptor/ trigger mechanism, yet cannot trigger germination in the absence of the gerD and ger $K$ gene products and further germinants. Spores of null mutants of $\operatorname{ger} A$ respond weakly to ALA with the GFK adjuncts, and this is dependent upon (at least) the ger $B$ and ger $K$ gene products (McCann, 1989). As GerK and GerD have been tentatively assigned the roles of glucose and fructose receptors respectively, it can be suggested that ger $B$ encodes the ASN receptor which may also act as the secondary ALA receptor proposed by Sammons et al. (1981). The demonstration that gerB is a homologue of the probable principal ALA receptor/trigger reinforces this argument.

It is not obvious why the ger $B$ products cannot stimulate germination in their own tight. It now seems likely that ALA and AGFK germination occur by a more similar mechanism than could previously have been suggested on the basis of genetical and physiological evidence and it is therefore proposed that there are two similar types of germinant receptors in the spore. One may involve GLU and FRU receptors which are facultatively required to improve the response to ALA alone mediated by the ger $A$ gene products. The other could have the GLU and FRU receptors working in obligate conjunction with an amino acid receptor, which is less fastidious than the ALA receptor and encoded by ger $B$. This model is summarized in Fig. 6. Whilst this model is novel in terms of the spatial arrangement and interactions between germination proteins that it implies, the mechanisms by which the germination apparatus function are still unknown and are the subject of our continuing enquiry.

\section{ACKNOWLEDGEMENTS}

The screening work was done by B. M.C. in the laboratories of Professor D. Karamata in Lausanne, and we are most grateful to him and members of his group for their kindness and hospitality. We would also like to thank Dr A. Moir and Professor C. M. Thomas and his group for much advice and many useful conversations, and Dr Moir for a critical reading of the manuscript. B. M.C. was an SERC postgraduate student. This work was completed with SERC research grant GR/H09164 to Dr Moir.

\section{REFERENCES}

Anagnostopoulos, C. \& Spizizen, J. (1961). Requirements for transformation in Bacillus subtilis. J Bacteriol 81, 741-746.

Chambers, S. P., Prior, S. E., Barstow, D. A. \& Minton, N. P. (1988). The pMTL nic-cloning vectors. 1. Improved pUC polylinker regions to facilitate the use of sonicated DNA for nucleotide sequencing. Gene 68, 139-149.

Devereux, J., Haeberli, P. \& Smithies, O. (1984). A comprehensive set of sequence analysis programmes for the VAX. Nucleic Acids Res 12, 387-395.

Fajardo-Cavazos, P., Tovar-Rojo, F. \& Setlow, P. (1991). Effect of promoter mutations and upstream deletions on the expression of 
genes coding for small, acid-soluble spore proteins of Bacillus subtilis. J Bacteriol 173, 2011-2016.

Feavers, I. M., Miles, J. S. \& Moir, A. (1985). The nucleotide sequence of a spore germination gene (ger $A$ ) of Bacillus subtilis 168 . Gene 38, 95-102.

Feavers, I. M., Foulkes, J., Setlow, B., Sun, D., Nicholson, W., Setlow, P. \& Moir, A. (1990). The regulation of transcription of the ger $A$ spore germination operon of Bacillus subtilis. Mol Microbiol 4, 275-282.

Fort, P. \& Errington, J. (1985). Nucleotide sequence and complementation analysis of a polycistronic sporulation operon, spo $V A$, in Bacillus subtilis. J Gen Microbiol 131, 1091-1105.

Higgins, C. F., Gallagher, M. P., Hyde, S. C., Mimmack, M. L. \& Pearce, S. R. (1990). Periplasmic binding protein-dependent transport systems: the membrane associated components. Pbilos Trans $R$ Soc London B326, 353-365.

Hiles, I. D., Gallagher, M. P., Jamieson, D. J. \& Higgins, C. F. (1987). Molecular characterization of the oligopeptide permease of Salmonella typhimurium. J Mol Biol 195, 125-142.

Irie, R., Okamoto, T. \& Fujita, Y. (1982). A germination mutant of Bacillus subtilis deficient in response to glucose. J Gen Appl Microbiol 28, 345-354.

Irie, R., Okamoto, T. \& Fujita, Y. (1986). Characterization and mapping of Bacillus subtilis gerD mutants. J Gen Appl Microbiol 32, 303-315.

Kyte, J. \& Doolittle, R. F. (1982). A simple method for displaying the hydropathic character of a protein. J Mol Biol 157, 105-132.

Mandel, M. \& Higa, A. (1970). Calcium-dependent bacteriophage infection. J Mol Biol 53, 159-162.

Maniatis, T., Fritsch, E. F. \& Sambrook, J. (1982). Molecular Cloning. A Laboratory Manual. Cold Spring Harbor, New York: Cold Spring Harbor Laboratory.

Margot, P. (1992). Génétique des autolysines de Bacillus subtilis. PhD thesis, Université de Lausanne, Switzerland.

Mauël, C., Young, M., Margot, P. \& Karamata, D. (1989). The essential nature of teichoic acids in Bacillus subtilis as revealed by insertional mutagenesis. Mol \& Gen Genet 215, 388-394.

McCann, K. P. (1989). A study of gerB and gerK spore germination mutants of Bacillus subtilis 168. MSc thesis, University of Birmingham, UK.

Moir, A. \& Smith, D. A. (1990). The genetics of bacterial spore germination. Annu Rev Microbiol 44, 531-553.

Moir, A., Lafferty, E. \& Smith, D. A. (1979). Genetic analysis of spore germination mutants of Bacillus subtilis: correlation of map position with phenotype. J Gen Microbiol 111, 165-180.
Piggot, P. J.s Amjad, Wu J.-J., Sandoval, H. \& Castro, J. (1990). Genetic and physical maps of Bacillus subtilis 168. In Molecular Biological Methods for Bacillus, pp. 493-543. Edited by C. R. Harwood \& S. M. Cutting. Chichester: Wiley.

Sammons, R. L., Moir, A. \& Smith, D. A. (1981). Isolation and properties of spore germination mutants of Bacillus subtilis 168 deficient in the initiation of germination. $J$ Gen Microbiol 124, 229-241.

Sammons, R. L., Slynn, G. M. \& Smith, D. A. (1987). Genetic and molecular studies on ger $M$, a new developmental locus of Bacillus subtilis. J Gen Microbiol 133, 3299-3312.

Sorokin, A., Zumstein, E., Azevedo, V., Ehrlich, S. D. \& Serrer, P. (1993). The organization of the Bacillus subtilis 168 chromosome region between the spo $V A$ and ser $A$ genetic loci, based on sequence data. Mol Microbiol 10, 385-395.

Stewart, G. C. \& Bott, K. (1983). DNA sequence of the tandem ribosomal-RNA promoter for B. subtilis operon $\mathrm{rrnB}$. Nucleic Acids Res 11, 6289-6300.

Venkatasubramanian, P. \& Johnstone, K. (1989). Biochemical analysis of the Bacillus subtilis 1604 spore germination response. J Gen Microbiol 135, 2723-2733.

Yamaguchi, K., Yu, F. \& Inouye, M. (1988). A single amino-acid determinant of the membrane localization of lipoproteins in Escherichia coli. Cell 53, 423-432.

Yamamoto, J., Shimizu, M. \& Yamane, K. (1991). Molecular cloning and analysis of nucleotide sequence of the Bacillus subtilis lys $A$ gene region using Bacillus subtilis phage vectors and a multicopy plasmid, pUB110. Agric Biol Chem 55, 1615-1626.

Yon, J. R., Sammons, R. L. \& Smith, D. A. (1989). The cloning and sequencing of the gerD gene of Bacillus subtilis. J Gen Microbiol 135, 3413-3445.

Young, M., Mauěl, C., Margot, P. \& Karamata, D. (1989). Pseudoallelic relationship between non-homologous genes concerned with biosynthesis of polyglycerol phosphate and polyribitol phosphate teichoic acids in Bacillus subtilis strains 168 and W23. Mol Microbiol 3, 1805-1812.

Zuberi, A. R., Feavers, I. M. \& Moir, A. (1985). Identification of three complementation units in the ger $A$ spore germination locus of Bacillus subtilis. J Bacteriol 162, 756-762.

Zuberi, A. R., Feavers, I. M. \& Moir, A. (1987). The nucleotide sequence and gene organization of the ger $A$ spore germination operon of Bacillus subtilis 168. Gene 162, 756-762.

Received 1 June 1993; revised 27 September 1993; accepted 6 October 1993. 